\title{
Rapid production of large-area, transparent and stretchable electrodes using metal nanofibers as wirelessly operated wearable heaters
}

\author{
Jiuk Jang ${ }^{1}$, Byung Gwan Hyun ${ }^{1}$, Sangyoon $\mathrm{Ji}^{1}$, Eunjin Cho, Byeong Wan An, Woon Hyung Cheong \\ and Jang-Ung Park
}

A rapidly growing interest in wearable electronics has led to the development of stretchable and transparent heating films that can replace the conventional brittle and opaque heaters. Herein, we describe the rapid production of large-area, stretchable and transparent electrodes using electrospun ultra-long metal nanofibers (mNFs) and demonstrate their potential use as wirelessly operated wearable heaters. These mNF networks provide excellent optoelectronic properties (sheet resistance of $\sim 1.3 \Omega$ per sq with an optical transmittance of $\sim 90 \%$ ) and mechanical reliability ( $90 \%$ stretchability). The optoelectronic properties can be controlled by adjusting the area fraction of the mNF networks, which also enables the modulation of the power consumption of the heater. For example, the low sheet resistance of the heater presents an outstanding power efficiency of $0.65 \mathrm{~W} \mathrm{~cm}^{-2}$ (with the temperature reaching $250^{\circ} \mathrm{C}$ at a low DC voltage of $4.5 \mathrm{~V}$ ), which is $\sim 10$ times better than the properties of conventional indium tin oxide-based heaters. Furthermore, we demonstrate the wireless fine control of the temperature of the heating film using Bluetooth smart devices, which suggests substantial promise for the application of this heating film in next-generation wearable electronics.

NPG Asia Materials (2017) 9, e432; doi:10.1038/am.2017.172; published online 8 September 2017

\section{INTRODUCTION}

Transparent conductive electrode (TCE) materials, such as graphene, carbon nanotubes (CNTs), metal oxides and metal nanowires, have been used in various fields, ${ }^{1-3}$ and their application as transparent heaters has also attracted attention. Transparent heaters, using the resistive heating of transparent conductive films, have been used extensively to control temperature for the stable operation of various devices, ${ }^{4,5}$ such as defogging windows and mirrors in cars, ${ }^{6}$ enhancing the sensitivity of gas sensors, ${ }^{5}$ and providing thermal treatment for arthritis and cancer. ${ }^{7}$ Optical transmittance and sheet resistance $\left(R_{\mathrm{S}}\right)$, which are the main factors that determine the properties of transparent conductive films, are also crucial criteria for the performance of transparent heaters. In particular, a low $R_{\mathrm{s}}$ value and good transparency in the range of visible light wavelengths are highlighted as some of the virtues of transparent heaters since they are directly related to the enhancement of power efficiency. Although indium tin oxide (ITO) is a commonly used transparent and conductive material for these heaters because of its good optoelectronic properties ( $<\sim 80 \Omega$ per sq with a transmittance of $90 \%$ at $550 \mathrm{~nm}$ ), ${ }^{8}$ its brittleness under mechanical strain, such as bending or stretching, limits its potential application in stretchable and wearable electronic devices. ${ }^{9}$ In addition, the low thermal conductivity of ITO can lead to slow heating and cooling rates. ${ }^{10}$ Because of these disadvantages of ITO, the use of low-dimensional and stretchable conductive materials, such as graphene, ${ }^{11-13} \mathrm{CNTs},{ }^{14,15}$ metal nanowires $(\mathrm{mNWs})^{7,16-18}$ and serpentine patterns of metal films, ${ }^{19,20}$ has been studied extensively in the search for stretchable heaters. However, carbon-based, transparent heaters require high operation voltages because of their relatively high $R_{\mathrm{s}}$ values without doping ( $>\sim 250 \Omega$ per sq), which can degrade their power efficiency. Therefore, additional chemical treatments to enhance $R_{\mathrm{s}}$ are required for the desired temperature ranges. ${ }^{21}$ Although stretchable serpentine patterns of metal films can present relatively low resistances, the opaqueness of these metals is disadvantageous for producing heaters with high transparency, and an additional patterning process such as photolithography is required to form the serpentine structure. ${ }^{22}$ Although the $R_{\mathrm{s}}$ values of $\mathrm{mNW}$ networks ( $>\sim 20 \Omega$ per sq) for transmittances of $>90 \%$ are comparable to those of ITO, and their mechanical deformability is remarkable due to their mesh-like percolating structures, ${ }^{23,24}$ their low aspect ratios and high inter-NW junction resistances limit the maximization of their overall electrical performance. ${ }^{25}$ Charge transport occurs along one-dimensional (1D) metallic pathways, and the

School of Materials Science and Engineering, Wearable Electronics Research Group, Ulsan National Institute of Science and Technology (UNIST), Ulsan Metropolitan City, Republic of Korea

1These authors contributed equally to this work.

Correspondence: Professor J-U Park, School of Materials Science and Engineering, Wearable Electronics Research Group, Ulsan National Institute of Science and Technology (UNIST), Ulsan Metropolitan City 44919, Republic of Korea.

E-mail: jangung@unist.ac.kr

Received 12 April 2017; revised 16 June 2017; accepted 10 July 2017 
ultra-long lengths of conductive nanowires or nanofibers facilitate the reduction of $R_{\mathrm{S}}$ by minimizing the number of junctions between the metal fibers. Therefore, conductive networks of $1 \mathrm{D}$ nanomaterials with ultra-high aspect ratios could be a solution to overcome the current limitations of NWs for stretchable, transparent heaters. ${ }^{26-32}$ In this respect, percolating networks of ultra-long, 1D metal nanotroughs can be fabricated by depositing a metal onto a sacrificial polymeric substrate, electrospinning the polymer, and then selectively removing the polymer to produce a free-standing, nanofiber web as the template. ${ }^{6,26-28}$ Although the geometry of ultra-long nanotroughs can provide low $R_{\mathrm{s}}$ levels ( $<17 \Omega$ per sq for transmittance $>90 \%$ ), it requires multiple processing steps that can increase the overall fabrication cost, and it is difficult to produce large-area nanotrough films. ${ }^{26}$ Thus, to overcome these limitations, novel methods are required to form ultra-long metal nanofibers directly.

Herein, we report the use of a rapid electrospinning process using a roll that can directly form $1 \mathrm{D}$, ultra-long Ag nanofibers (AgNFs) as a large-area continuous network for transparent, stretchable electrodes and heaters. This electrospinning process is relatively simple and lowcost, since AgNFs can be formed directly on a flexible sheet while minimizing the waste of functional inks (suspensions of Ag nanoparticles). Specifically, this process, which is based on a roll collector, enables the rapid production of a transparent, conductive film that is composed of a large-area AgNF network. This electrode film exhibits superb optoelectronic characteristics $\left(R_{\mathrm{s}}\right.$ of $\sim 1.3 \Omega$ per sq with an optical transmittance of $\sim 90 \%$ in the visible regime) and outstanding mechanical properties $(90 \%$ stretchability and a minimum bending radius of curvature of $70 \mu \mathrm{m})$. A transparent and stretchable heater can be produced by the Joule heating of this film, that is, converting the energy of the electric current into heat. Adjusting the area fraction of the AgNF network by tuning the duration of the electrospinning process allows us to control the optoelectronic properties of the film, and this adjustment also enables the modulation of the temperature range and power consumption of the heater. The maximum temperature of this heating film is $\sim 250^{\circ} \mathrm{C}$ on a polyimide (PI) sheet; however, it can be increased to more than $600{ }^{\circ} \mathrm{C}$ on a quartz substrate. The temperature distribution of this heater under mechanical deformation is uniform because of its good flexibility and stretchability. In contrast with the conventional heating films based on ITO, the heating and cooling rates of this AgNF heater are relatively rapid due to the high thermal conductivity of Ag. Furthermore, the heater's integration with a battery and Bluetooth module demonstrates that it can serve as a portable, transparent heater with precise, wireless temperature control using a smartphone. We believe that the formation of these transparent, stretchable electrodes and heaters using ultra-long AgNF networks presents great opportunities for nextgeneration, wearable electronics.

\section{MATERIALS AND METHODS}

Fabrication of ultra-long AgNF random network electrodes

We used an electrospinning process to fabricate a continuous network of $\mathrm{Ag}$ nanofibers (AgNFs) with an average diameter of $338 \pm 35 \mathrm{~nm}$ using a suspension of $\mathrm{Ag}$ nanoparticles (NPK, Korea; average diameter: $40 \pm 5 \mathrm{~nm}$; solvent: ethylene glycol; concentration $=50 \mathrm{wt} . \%$ ) as an ink. The electrospinning height was $15 \mathrm{~cm}$, the applied voltage between the nozzle tip and the ground was $11.5 \mathrm{kV}$, and the inner and outer diameters of the nozzle were 0.33 and $0.64 \mathrm{~mm}$, respectively. The environmental temperature and relative a

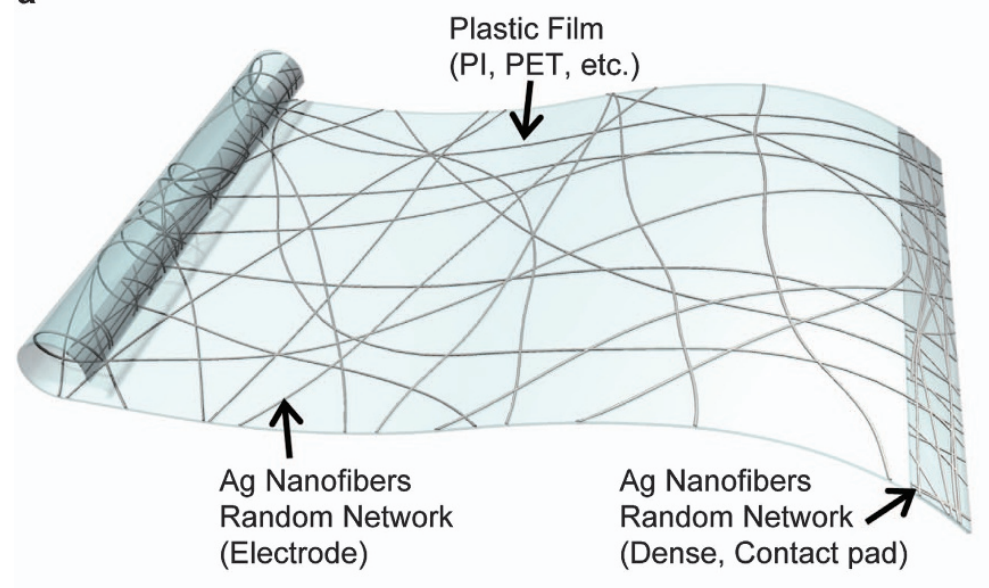

C

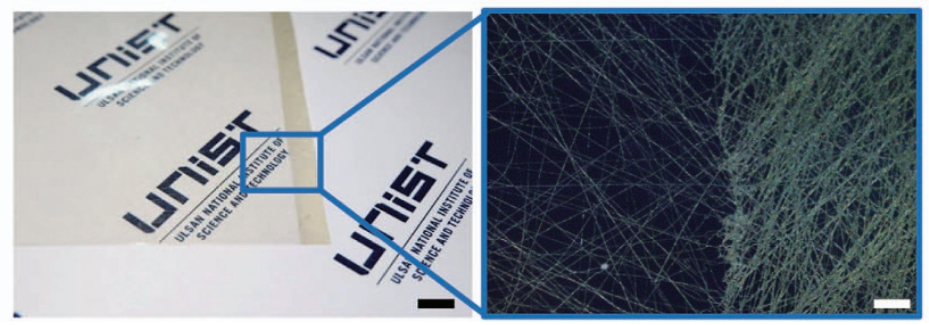

b

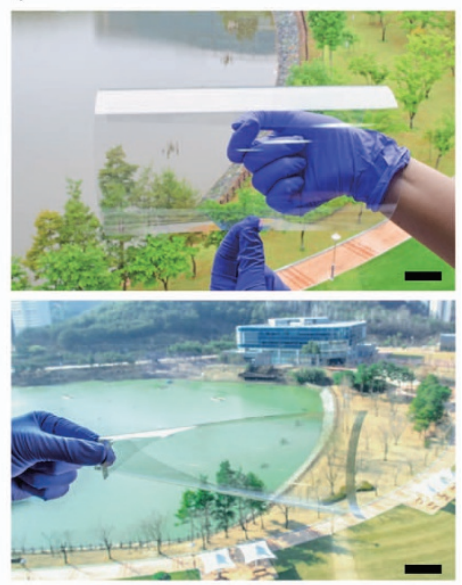

d

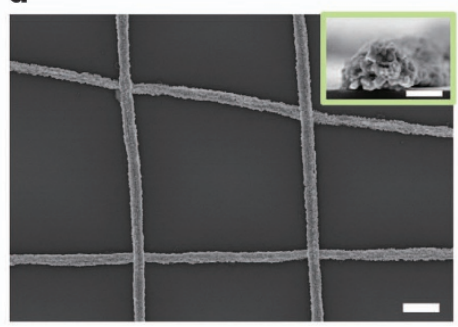

Figure 1 Characterization of a stretchable, transparent and large-area AgNF heater. (a) Schematic illustration of the stretchable and transparent heater composed of a AgNF random network. (b) Photographs of a stretchable and transparent electrode and large-area heater using a AgNF random network on a PET film. The scale bar represents $3 \mathrm{~cm}$. (c) A photograph (left) and magnified optical micrograph (right) of the border between the electrode and the contact pad. The left scale bar represents $5 \mathrm{~mm}$, and the right scale bar represents $20 \mu \mathrm{m}$. (d) Scanning electron microscope image of a AgNF random network. The scale bar represents $1 \mu \mathrm{m}$. The inset shows the magnified image of a single AgNF. The scale bar represents $200 \mathrm{~nm}$. 
humidity were $17^{\circ} \mathrm{C}$ and $4 \%$, respectively. The continuous production of the large-area electrode was realized using a drum collector (width: $30 \mathrm{~cm}$, radius: $6 \mathrm{~cm}$, rotating speed: 2000 r.p.m.). The electrospun fibers were annealed at $150^{\circ}$ C for $30 \mathrm{~min}$ in air (relative humidity: $\sim 25 \%$ ). The photonic annealing was carried out using a xenon lamp system (UNILAM, Ulsan Metropolitan City, Korea) in which a xenon lamp was equipped with a bandpass filter for a broad spectrum of 400 to $1100 \mathrm{~nm}$. The dimensions of this lamp were $10 \mathrm{~cm}$ in width and $30 \mathrm{~cm}$ in length, and the films could be moved in the longitudinal direction using a roll. This xenon flash lamp was located $10 \mathrm{~cm}$ away from the substrate stage with a water cooling system. The total energy delivered was $22.4 \mathrm{~J} \mathrm{~cm}^{-2}$, the width of light of the output pulse was $5 \mathrm{~ms}$, and the gap between the pulses was $500 \mathrm{~ms}$. The substrates used in the experiment were 2-mm-thick quartz, $100-\mu \mathrm{m}$-thick PET film (Toray Industries Inc., Tokyo, Japan), and 50- $\mu \mathrm{m}$-thick PI film (Mitsubishi Corp., Tokyo, Japan). In particular, the PI film from Mitsubishi Corp. had a glass transition temperature $\left(T_{\mathrm{g}}\right)$ of $302^{\circ} \mathrm{C}$, and it could endure temperatures above the $T_{\mathrm{g}}$ of the PET film. Details about the fabrication method of the electrospun metal NF network onto the flexible substrate can be found elsewhere. ${ }^{26,28-31}$ As a transparent electrode, the low-density AgNF networks formed by electrospinning for $5 \mathrm{~s}$ exhibited a significantly low sheet resistance of $1.3 \pm 0.2 \Omega$ per sq with a transmittance of $90 \%$.

\section{Fabrication of heaters based on AgNFs, AgNWs and ITO}

To fabricate the AgNF heater, an additional electrospinning process was performed on the transparent and stretchable AgNF electrode. The heating region of the AgNF heater was shaded by a shadow mask during the process, and the rest of the region had a dense AgNF network, which led to very low sheet resistance (as shown in Figure 1a). The dense region at both ends acted as the connection points for the AgNF heater. We can operate the heater directly by applying a DC bias to both ends. To fabricate the AgNW heater, a commercial suspension of AgNWs (Nanopyxis Co. Ltd., average AgNW diameter of $30 \pm 5 \mathrm{~nm}$ and length of $25 \pm 5 \mu \mathrm{m}$, solvent: deionized water, concentration: $3 \mathrm{mg} \mathrm{ml}^{-1}$ ) was spin-coated for $30 \mathrm{~s}$ at 500 r.p.m. onto a PET film. To fabricate the ITO heater, an ITO film (thickness: $120 \mathrm{~nm}$ ) was deposited on a PET substrate using an RF sputter system (SRN-120, SORONA, Pyeongtaek, Korea). The AgNW-heater and ITO-heater exhibited sheet resistances of 10 and $50 \Omega$ per sq, respectively. The transmittance of both heaters was $\sim 90 \%$ in the visible regime. Cu-wire interconnects were attached to both ends of each heater using a silver epoxy (ELCOAT A-200, CANS, Tokyo, Japan), followed by epoxy curing for $10 \mathrm{~min}$ at $150{ }^{\circ} \mathrm{C}$ in air (relative humidity: 25\%).

\section{Integration of wireless controller devices}

The wireless temperature controller device was designed using open source electronic modules and coding. The device consisted of a microcontroller unit, Bluetooth module (Bluetooth Low Energy (BLE) 4.0), battery (240 mAh), and high-voltage and current controller unit, as shown in Figure 5a. We integrated all the components into a tiny single-circuit device, which was $2.4 \times 3.2 \mathrm{~cm}^{2}$ and $40 \mathrm{~g}$. A small temperature sensor (thermistor) was attached directly to the heater for real-time feedback.

\section{Bending and stretching tests}

For the bending test, a $2-\mu \mathrm{m}$-thick PI film, which was spin-casted from a PI precursor (poly(pyromellitic dianhydride-co-4,4'oxydianiline), Aldrich), was used as a substrate. The electrospun AgNF random network was directly formed on the substrate. After thermal annealing at $150{ }^{\circ} \mathrm{C}$ for $30 \mathrm{~min}$ in air (relative humidity: $\sim 25 \%$ ), gold contact pads were thermally deposited on the AgNF random network electrode. The samples were conformally wrapped on various objects that had a small radius of curvature, and changes in the relative resistance were measured using the two-point probe method (4200-SCS, Keithley, Cleveland, OH, USA). The bending-induced strain was calculated using the following equation:

$$
\varepsilon_{\text {bending }}=\frac{t_{\mathrm{s}}+t_{\mathrm{f}}}{2 r_{\mathrm{c}}}
$$

where $r_{\mathrm{c}}$ is the radius of curvature, and $t_{s}$ and $t_{\mathrm{f}}$ are the thicknesses of the substrate and film, respectively.
For the stretching experiment, AgNFs were electrospun on a poly(methyl methacrylate) (PMMA) sheet, followed by thermal annealing at $150{ }^{\circ} \mathrm{C}$ for $30 \mathrm{~min}$ in air (relative humidity: $\sim 25 \%$ ). After casting a polydimethylsiloxane (PDMS) layer on the AgNFs, the sample was soaked in acetone to dissolve the sacrificial PMMA layer, forming a stretchable AgNF electrode. The random network of AgNFs on the PDMS sheet was clamped by two fixtures connected to a current-voltage source meter system (4200-SCS, Keithley). It was stretched in the uniaxial direction using a mechanical apparatus. During the stretching test, both ends of the heater ( $12 \%$ of the total film area for each end) were anchored to the stretching stage, and $\sim 65 \%$ of the total area, except for the portion near the fixed area, was stretched. The stretch-induced strain was calculated using the following equation:

$$
\varepsilon_{\text {stretching }}=\frac{L_{\mathrm{s}}}{L_{0}} \times 100
$$

where $L_{\mathrm{s}}$ and $L_{0}$ are the length after stretching and before stretching, respectively.

\section{Characterization of AgNF random network electrodes}

The surface morphology of the AgNFs was observed using a scanning electron microscope (S4600, Hitachi, Tokyo, Japan), optical microscope (BX53, Olympus, Tokyo, Japan), high-resolution transmission electron microscope (HR-TEM) (JEM-2100F, JEOL, Tokyo, Japan) and atomic force microscope (Asylum Research, Goleta, CA, USA). The microstructure of the AgNFs was analyzed using X-ray diffraction (XRD) equipment (D8 Advance, Bruker, Billerica, MA, USA) and X-ray photoelectron spectroscopy (XPS) (ESCALAB 250XI, Thermo Fisher Scientific, Waltham, MA, USA), as well as HR-TEM. The four-point probe method was used for the sheet resistance measurement using a probe station with a Keithley 4200-SCS semiconductor parametric analyzer. The optical transmittance was measured using UV-Vis-NIR spectroscopy (Cary 5000 UV-Vis-NIR, Agilent, Santa Clara, CA, USA) with a diffuse reflectance accessory, and the transmittance of the substrate was used as a baseline. The transmittance of the substrate was excluded.

\section{Thermal characterization}

The DC bias was applied using a power supply (Keithley 2260B-30-72), and the temperature was measured using an LWIR camera (T650sc, FLIR Systems, Wilsonville, OR, USA). The temperature distribution was analyzed using the FLIR ResearchIR software (Research IR Max, FLIR Systems).

\section{RESULTS AND DISCUSSION}

\section{Fabrication of ultra-long AgNF random network electrodes}

Figure 1a shows a schematic illustration of the stretchable transparent electrode based on a AgNF random network, which was composed of Ag nanoparticles. In the first step of the fabrication process, a random network of AgNFs was collected onto a flexible and stretchable sheet such as PET, PI or PDMS using an electrospinning system with a roll collector. The plastic film substrate was wrapped on the outer surface of a grounded drum-shaped metallic collector (width: $30 \mathrm{~cm}$, radius: $6 \mathrm{~cm}$, rotating speed: 2000 r.p.m.), and a suspension of Ag nanoparticles (NPK, Korea, average diameter: $40 \pm 5 \mathrm{~nm}$, solvent: ethylene glycol, concentration $=50 \mathrm{wt} . \%$ ) was electrospun continuously onto the rotating substrate to form ultra-long AgNFs (inner nozzle size: $0.33 \mathrm{~mm}$, outer nozzle size: $0.64 \mathrm{~mm}){ }^{26-29,31,32}$ As shown in Supplementary Movie S1, this roll-based electrospinning system enabled the relatively rapid production of a transparent conductive film composed of a AgNF network with a large area (width of film: $30 \mathrm{~cm}$, production speed: $1.5 \mathrm{~m} \mathrm{~min}^{-1}$ in the film length). Compared with other printing methods (such as inkjet printing, electrohydrodynamic jet printing, imprinting, indirect electrospinninng and laserassisted printing) used to form transparent electrodes, ${ }^{11,26-29,33-35}$ this direct electrospinning of AgNFs is advantageous for the rapid production of large-area conductive films using a roll. After detaching this electrospun film from the drum collector, it was thermally annealed at $150{ }^{\circ} \mathrm{C}$ for $30 \mathrm{~min}$ in air (relative humidity: $\sim 25 \%$ ) to 
a

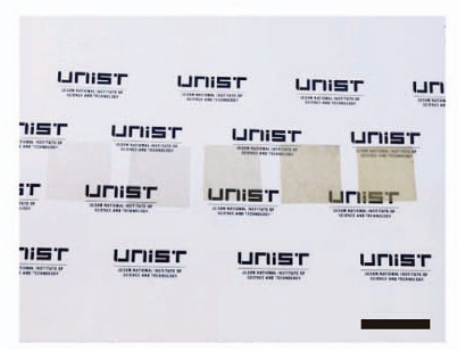

d

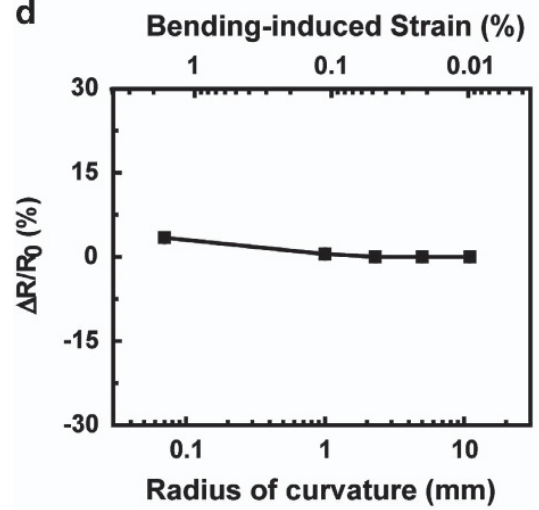

b

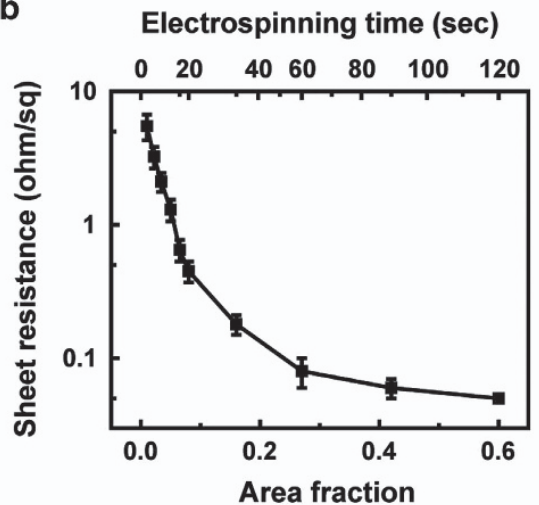

e

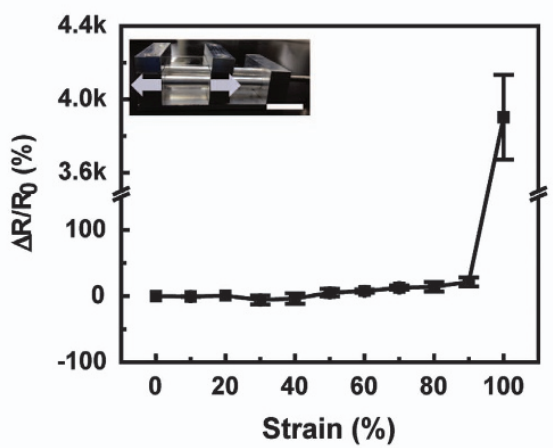

C

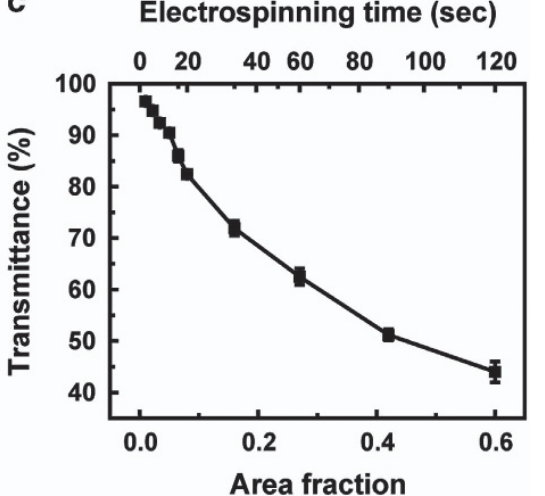

f

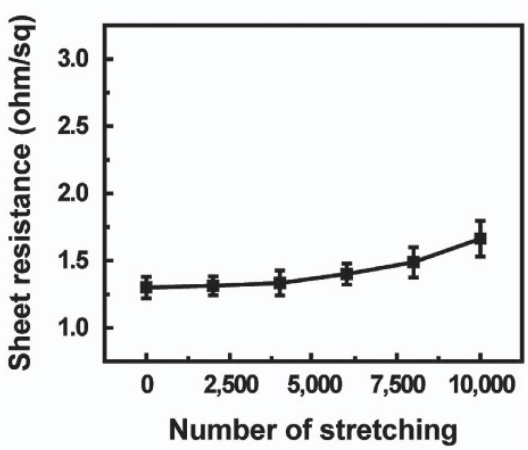

Figure 2 Optoelectronic and mechanical properties of a AgNF random network electrode. (a) A photograph of stretchable and transparent AgNF random network electrodes for area fractions of 0.05 (electrospinning time: $5 \mathrm{~s}$ ), 0.08 (20 s), 0.15 (30 s), 0.3 (60 s) and 0.6 (120 s) (from left to right). The scale bar represents $3 \mathrm{~cm}$. (b) The sheet resistance and (c) optical transmittance as a function of area fraction and electrospinning time. (d) The relative resistance change as a function of the radius of curvature and bending-induced strain. (e) The relative resistance change as a function of tensile strain. The inset shows the stretching direction. The scale bar represents $2 \mathrm{~cm}$. (f) The cyclic stretching test with applied strain ( 30\%).

coalesce the Ag nanoparticles into electrically conductive AgNFs with an average diameter of $338 \pm 35 \mathrm{~nm}$ (Supplementary Figure S1). This thermal annealing step did not break the AgNFs, and the single fibers were long enough to cross an A4-size sheet from one edge to the opposite edge without becoming disconnected. For a continuous rollto-roll process, we tested a photonic annealing method, as well as the thermal annealing method, and the optoelectronic properties of the AgNF electrodes using this photonic annealing were comparable with those of the thermally annealed samples (Supplementary Figure S2). In contrast to the slow thermal annealing, the relatively fast speeds of this photonic annealing process (in the range of $4.5-12 \mathrm{~m} \mathrm{~min}^{-1}$ by considering the lamp cooling time) are advantageous to the continuous production of AgNFs using a roll-to-roll system. The ultra-long lengths of the AgNFs can minimize the number of junctions between the 1D metal structures, which can lead to a significant reduction of $R_{\mathrm{s}}$, while maintaining large open spaces in the network for high transmittance. Additionally, these mesh-like, percolating networks facilitate their high stretchability. The energy of the electric currents that pass through these networks can radiate heat; hence, this transparent, conductive film can be used as a resistive heater. For the fabrication of this heater, relatively dense networks of AgNFs were additionally electrospun on both sides of this conductive film to provide two contact pads for applying the voltage, after screening off the central heating zone using a mask. Figure $1 \mathrm{~b}$ shows photographs of the transparent electrode and heater, where the random networks of AgNF were formed on an A4-sized PET sheet. Figure 1c shows a photograph and an optical micrograph of the border between the resistive heating zone (transparent part) and the contact pad (dense AgNF networks). Since all the conductive parts are composed of AgNFs, the heating zone and contact pad can be thermally annealed together in a single step. The surface morphology and roughness of a single AgNF and the AgNF network were also investigated using a scanning electron microscope and atomic force microscope (Figure 1d and Supplementary Figure S3). The TEM images and diffraction patterns show that aggregates of single-crystalline Ag nanoparticles (AgNPs) were annealed to form individual AgNFs and a negligible layer of carbon remained (Supplementary Figure S4). To further investigate the microstructures of AgNFs, including their crystallographic structure and elemental composition, we performed XPS and XRD analyses (Supplementary Figures S5 and S6). The large Ag $3 \mathrm{~d}_{5 / 2}$ sharp peak at $368.3 \mathrm{eV}$ indicates a large amount of silver with the dominant atomic percentage (over 95\%) as metallic Ag and negligible portions of silver oxides.

\section{Optical, mechanical and thermal characteristics of the AgNF} network electrode

The area fraction (or density) of the AgNF network, which can be controlled by the electrospinning time, is an important factor that is required to determine the $R_{\mathrm{s}}$ and the transmittance of the conductive film. For example, the photograph of the five samples in Figure 2a shows that the AgNF networks were formed with different area fractions on an identical PET sheet. A higher area fraction, which means a higher density of AgNFs, can be formed by electrospinning for a longer period (Supplementary Figures S7 and S8). Figure 2b and 
a
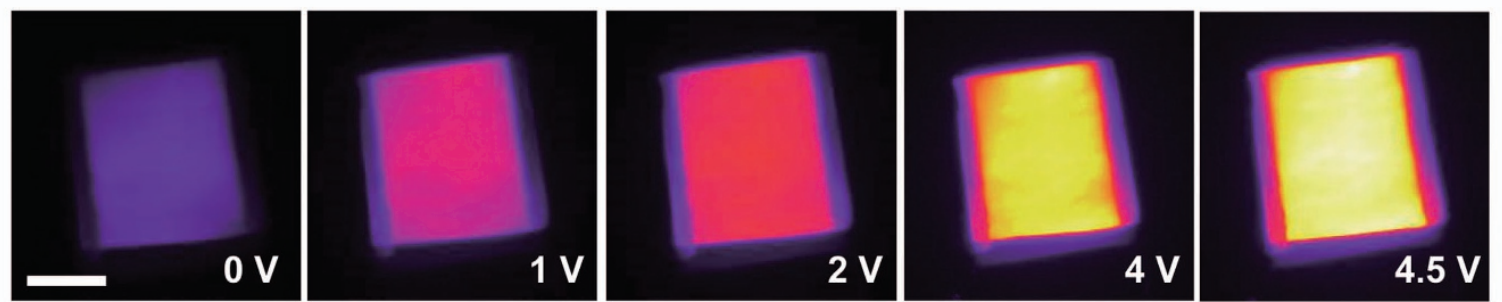

$260\left({ }^{\circ} \mathrm{C}\right)$

b
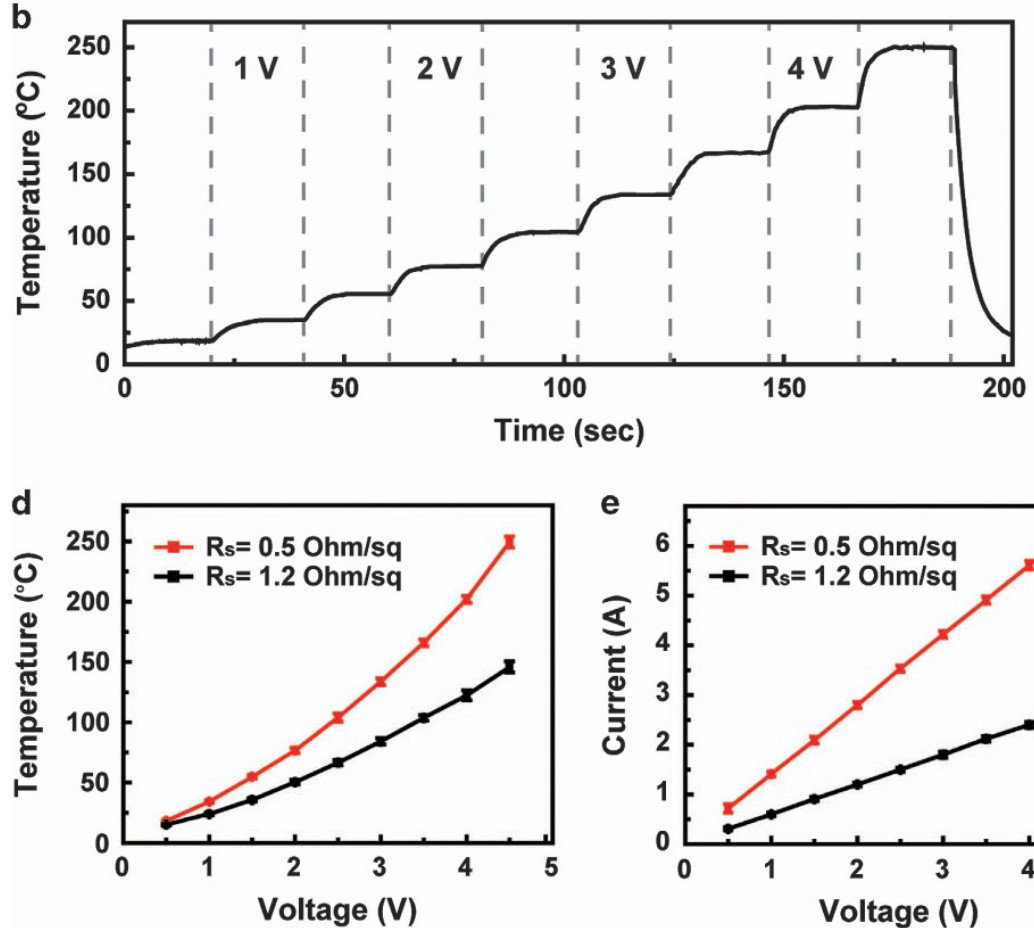

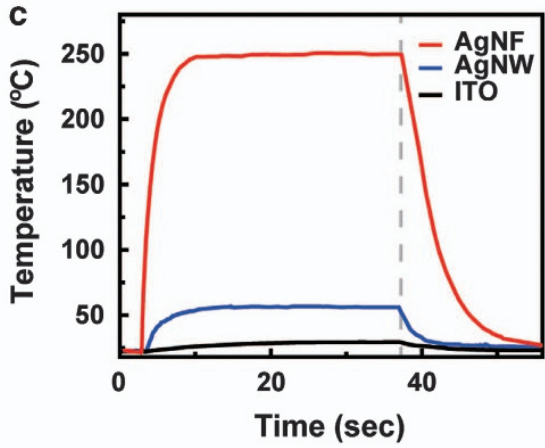

e

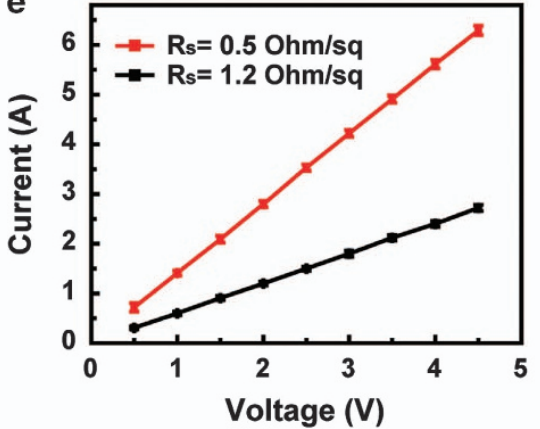

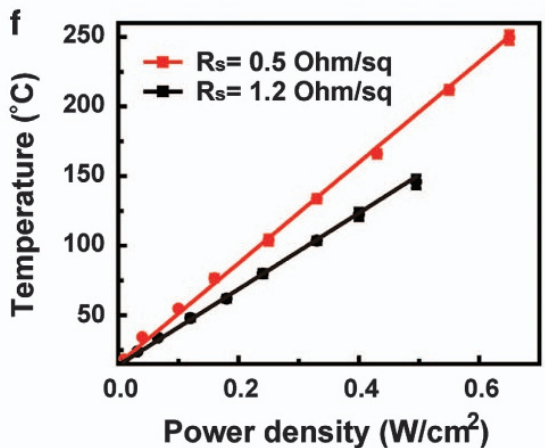

Figure 3 Characterization of heaters based on the AgNF network. (a) IR images of the heater for different applied voltages, 0 , $1,2,4$ and $4.5 \mathrm{~V}$ (from left to right). The scale bars represent $3 \mathrm{~cm}$. (b) The temperature evolution of the heater for stepwise increases in voltage from 0.5 to $4.5 \mathrm{~V}$. (c) The average temperature as a function of time for different materials (ITO, $\mathrm{R}_{\mathrm{s}} \approx 50 \Omega$ per sq; Ag NWs, $\mathrm{R}_{\mathrm{s}} \approx 10 \Omega$ per sq; and AgNFs, $\mathrm{R}_{\mathrm{s}} \approx 0.5 \Omega$ per sq) at $4.5 \mathrm{~V}$. (d) The temperature as a function of voltage and (e) the $I-V$ characteristics of the heater during operation and depending on the sheet resistance. (f) The temperature as a function of the input power density for the heater at different sheet resistances.

c present the $R_{\mathrm{s}}$ and transmittance of the AgNF film as a function of the area fraction. Both the $R_{\mathrm{S}}$ and transmittance decrease as the area fraction increases. As a transparent electrode, the low-density AgNF networks formed by electrospinning for $5 \mathrm{~s}$ (area fraction: 0.05) exhibited a significantly low $R_{\mathrm{s}}$ value of $1.3 \pm 0.2 \Omega$ per sq with a transmittance of $90 \%$. This $R_{\mathrm{s}}$ value was significantly lower than those of other transparent electrodes, such as ITO $\left(R_{\mathrm{s}}>\sim 50 \Omega\right.$ per sq), undoped CVD-synthesized graphene $\left(R_{\mathrm{s}}>\sim 250 \Omega\right.$ per sq) or a network of mNWs $\left(R_{\mathrm{s}}>\sim 20 \Omega\right.$ per sq). In addition, the $R_{\mathrm{s}}$ value of the AgNF electrode can be further decreased to $<0.05 \pm 0.001 \Omega$ per sq for a transmittance of $<44 \%$ by increasing the area fraction above 0.6 (electrospinning time: $120 \mathrm{~s}$ ), making the AgNF electrode suitable for use as a highly conductive, semi-transparent electrode.

Outstanding mechanical robustness against bending and stretching, as well as remarkable optoelectronic properties, are essential prerequisites for applications in wearable electronics. Figure $2 \mathrm{~d}$ shows the relative changes in the resistance of the AgNF network electrode as a function of the radius of curvature and the corresponding bendinginduced strain $(\varepsilon)$. For this measurement, the electrode film was wrapped onto the curvilinear surfaces of various cylindrical supports with different radii of curvature. A negligible change in resistance was detected even when the electrode was bent to a radius of curvature as small as $70 \mu \mathrm{m}(\varepsilon<1.7 \%)$, which indicates its superb flexibility. The stretchability of the AgNF network electrode was measured by embedding AgNFs inside PDMS. The fabrication procedure is described in detail in the experimental section (Supplementary Figure S9), and these embedded AgNFs did not delaminate from PDMS with stretching. The sample was cut to prove that the structure was embedded inside the PDMS substrate. Optical micrographs of the cut sample (as the top-view and cross-section view) are shown in Supplementary Figure S10. Figure 2e shows that stretching this sample electrode up to $90 \%$ in strain resulted in a slight (21\%) increase in resistance. The stretchability of the AgNF electrode is significantly better than that of ITO (maximum tensile strain $<1 \%$ ) ${ }^{36}$ or planar graphene $(<5 \%),{ }^{37}$ and it is comparable to the stretchability of $\mathrm{mNW}$ networks $(>80 \%) .{ }^{24}$ During the electrospinning process, the whipping of a continuous jet results in the formation of random radial web-like geometries of fibers on a film. The ultra-long length of the AgNFs and their random network (similar to radial spider webs) contributes to the superb mechanical stretchability of AgNF films. ${ }^{38}$ Furthermore, to observe the durability against repetitive stretching and releasing, the $R_{\mathrm{s}}$ value of this AgNF sample was measured during cyclic 
stretching (10000 cycles of 30\%-strain stretching at the rate of $\left.2.54 \mathrm{~mm} \mathrm{~s}^{-1}\right)$. Since the stretching range of human skin is below $30 \%$ in strain, ${ }^{22}$ the mechanical durability in this strain range is important for applications in epidermal electronic devices. As plotted in Figure $2 \mathrm{f}$, the $R_{\mathrm{s}}$ value remained nearly constant throughout this test (with a relative increase of $<25 \%$ ), which explains the outstanding reliability of the AgNF network electrode against repetitive stretching.

Thermal characteristics of a AgNF heater

The joule heating of a AgNF network electrode can form a stretchable, invisible heater. Figure 3 shows the thermal characterizations of this heater. A AgNF random network with an $R_{\mathrm{s}}$ value of $0.5 \Omega$ per sq (the area fraction: 0.07 ) for the central heating zone (size: $5 \times 5 \mathrm{~cm}^{2}$ ) was prepared on a $50-\mu \mathrm{m}$-thick transparent polyimide film (Mitsubishi Corp., $T_{\mathrm{g}}=302^{\circ} \mathrm{C}$ ). Both ends of this heating film were connected to a voltage supplier after selectively forming additional dense networks of AgNFs at the ends to act as contact pads. The temperature distributions of this heating film at different DC voltages were observed using an infrared (IR) camera (FLIR Systems) (Figure 3a).
The temperature increased with the applied DC bias, and the temperature distribution in the heating zone was uniform (Supplementary Table S1). The time-dependent change in the average temperature of this AgNF heater is plotted in Figure 3b. The input DC bias was increased to $4.5 \mathrm{~V}$ in increments of $0.5 \mathrm{~V}$ every $20 \mathrm{~s}$, which increased its average temperature to $249.5( \pm 5.8){ }^{\circ} \mathrm{C}$. Supplementary Figure S11 presents the relationship between the resistance and temperature of AgNF heaters with different sheet resistances. The polyimide film began to deform when the temperature exceeded $300^{\circ} \mathrm{C}$, which is the glass transition temperature of the film, at input voltages above $4.5 \mathrm{~V}$. However, when a quartz substrate was used instead of a polyimide substrate, the maximum temperature was $350^{\circ} \mathrm{C}$ without any passivation (Supplementary Figure S12), and this can be increased further to more than $600{ }^{\circ} \mathrm{C}$ at higher input voltages by depositing a passivation layer of $\mathrm{SiO}_{2}$ (thickness $>500 \mathrm{~nm}$ ) on the AgNF network to retard the oxidation of Ag in air (Supplementary Figure S13). As the thermal annealing temperature increased up to $600^{\circ} \mathrm{C}$, the surface of the AgNFs became smoother by further agglomeration of the Ag nanoparticles that form the AgNFs; however, a

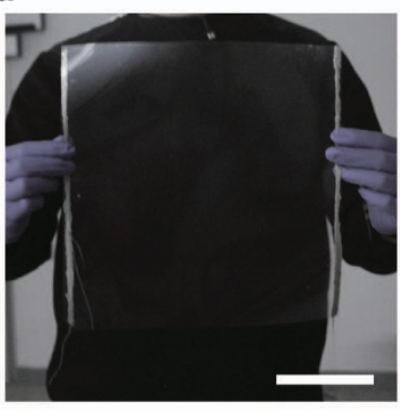

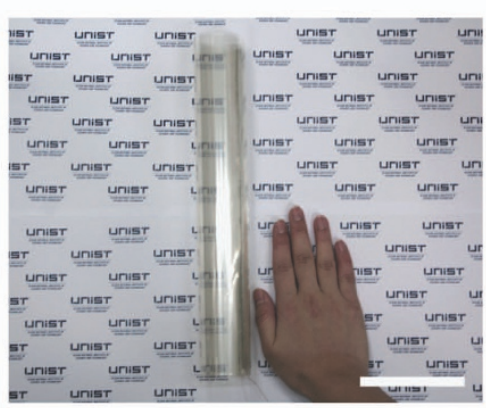

b

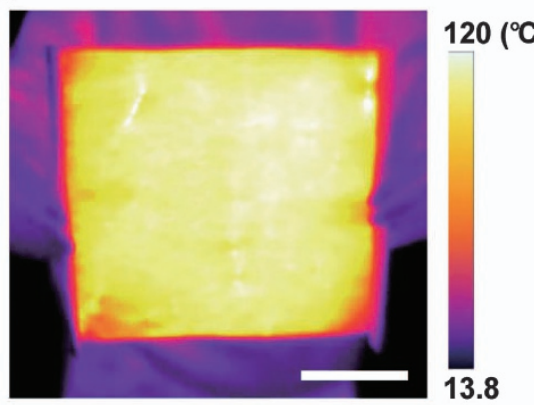

C
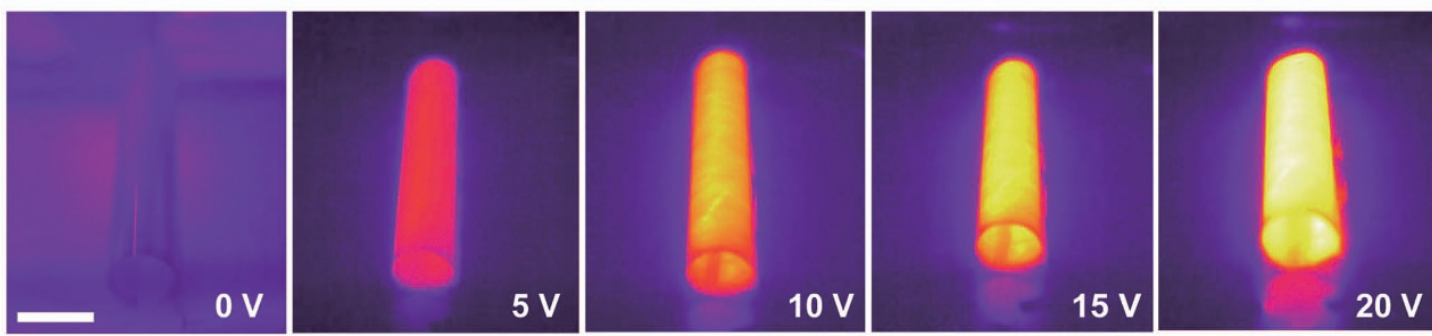

$260\left({ }^{\circ} \mathrm{C}\right)$

d

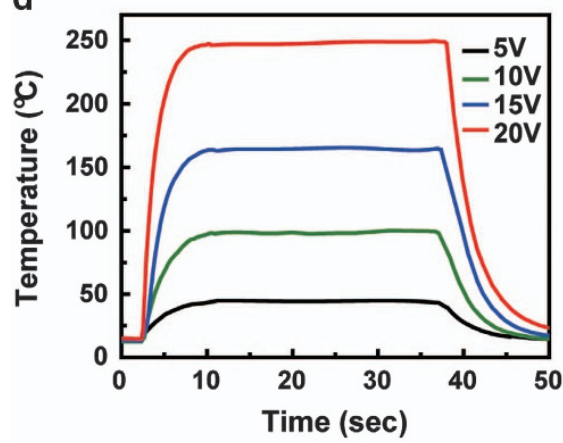

e

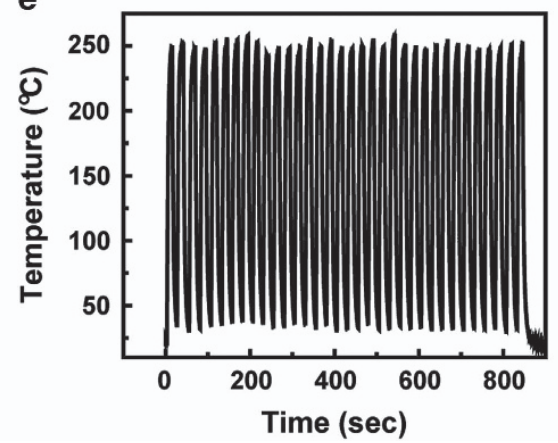

$\mathbf{f}$

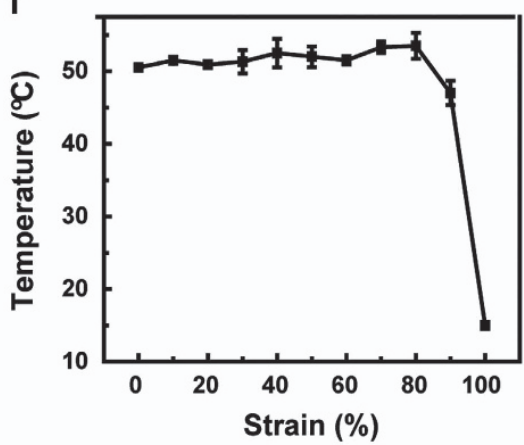

Figure 4 Demonstration of a large-area stretchable AgNF heater. (a) Photographs of the large-area heater before rolling (left) and the rolled heater (right). The scale bars represent $10 \mathrm{~cm}$. (b) An IR image of the large-area heater before rolling. The scale bars represent $10 \mathrm{~cm}$. (c) IR images of the rolled heater for different applied voltages, $0,5,10,15$ and $20 \mathrm{~V}$ (from left to right). The scale bars represent $5 \mathrm{~cm}$. (d) The temperature as a function of time for the rolled heater for different applied voltages (5, 10, 15 and $20 \mathrm{~V}$ ). (e) The cyclic thermal stability test of the AgNF heater. The plot shows the average temperature as a function of time. (f) The temperature as a function of applied strain. 
the fiber structure was maintained without disconnections, as shown in Supplementary Figure S14.

Figure 3c compares the properties of this AgNF heater with those of other transparent heaters based on ITO $\left(R_{\mathrm{s}} \approx 50 \Omega\right.$ per sq) and AgNWs ( $\sim 10 \Omega$ per sq). All of these different heaters were fabricated on polyimide substrates without a passivation layer, and they had the same optical transmittance ( $83 \%)$. The relatively low $R_{\mathrm{s}}(0.5 \Omega$ per sq) of this AgNF heater enabled it to pass relatively large currents at an identical input bias, resulting in its effective consumption of power. For example, when a DC voltage of $4.5 \mathrm{~V}$ was applied, the saturated temperature of the AgNF heater $\left(250^{\circ} \mathrm{C}\right)$ was significantly higher than those of the ITO heater $\left(29^{\circ} \mathrm{C}\right)$ and the AgNW heater $\left(54^{\circ} \mathrm{C}\right)$. The power efficiency of this AgNF-heater was $0.65 \mathrm{~W} \mathrm{~cm}^{-2}$, which was $\sim 10$ times better than the conventional ITO-based heaters. As presented in Figure $3 c$, the heating and cooling rates of this AgNF heater were relatively fast (reaching the saturated temperature within $10 \mathrm{~s}$ ) due to its low $R_{\mathrm{s}}$. This was in contrast to the rates of the other transparent heaters, indicating the advantage of this AgNF heater for potential application in rapidly heating or cooling films for windows, heat-distributing transparent layers, or thermal interface materials for transparent displays. Figure $3 \mathrm{~d}$ and e show the temperatures and currents of two AgNF heaters with different $R_{\mathrm{s}}$ values as a function of the applied DC voltage. These plots can be used to determine the relationship between the $R_{\mathrm{S}}$ value and the Joule heating performance. The current increased linearly with the input voltage for both AgNF heaters, and the temperature was approximately proportional to the square of the voltage. Figure $3 \mathrm{f}$ shows that the temperature of these two heaters increased linearly with the density of the input power. The slope of the best-fit straight line in Figure $3 \mathrm{f}$ represents the heating efficiency (that is, thermal resistance) of the heater. ${ }^{9,39}$ The heater that had a lower $R_{\mathrm{s}}$ value had a steeper slope, implying better heating efficiency. Therefore, the remarkably low $R_{\mathrm{s}}$ value of the AgNF heater was advantageous in reducing its thermal resistance for the efficient transduction of electrical energy into heat with lower power consumption.

\section{Demonstration of a large-area, flexible and transparent AgNF heater}

The roll-based electrospinning system can enlarge the size of this transparent and flexible heater using the AgNF network. As an example, Figure 4a shows a AgNF heater with a size of $30 \times 30 \mathrm{~cm}^{2}$ ( $R_{s}: 0.5 \Omega$ per sq, transmittance: $83 \%$, substrate: $50-\mu \mathrm{m}$-thick PI). The width of the drum collector in our electrospinning setup was limited and could not enlarge the width of this heater further. This large-area flexible heater can be rolled so that it is portable. Figure $4 \mathrm{~b}$ shows the thermal distribution of the large-area AgNF heater, exhibiting an average temperature of $103.8 \pm 4.1^{\circ} \mathrm{C}$. Supplementary Movie S2 presents demonstrations of the large-area flexible AgNF heater. As shown in the movie clip, the temperature of the heater was uniform during bending. To enhance the reliability of this large-area heater, the entire heating zone of the AgNF network (except the two contact pads) on the PI substrate was laminated with a $50-\mu$ m-thick PI film as a passivation layer using a large-area laminator (GMP, EXCELAMPLUS 685DCRSE). This plastic cover layer can prevent the permeation of oxygen gas and moisture to retard the oxidation of $\mathrm{Ag}$, and this lamination did not degrade the $R_{\mathrm{s}}$ value of the AgNF network. Figure $4 \mathrm{c}$ presents real-time IR images of this large-area heater in the rolled shape (radius of curvature: $1.5 \mathrm{~cm}$ ), indicating a uniform temperature distribution even during mechanical bending. The bending-induced temperature change is plotted in Supplementary Figure S15. For this measurement, the AgNF heater was wrapped on the outer surfaces of cylindrical supports with different radii of curvature. The temperature change was negligible even when the heater was bent to a radius of curvature as small as $70 \mu \mathrm{m}(\varepsilon<1.7 \%)$. Figure $4 \mathrm{~d}$ shows the corresponding temperature profiles of this heater at different input voltages (DC). The temperature of this heater was saturated at $250^{\circ} \mathrm{C}$ at $20 \mathrm{~V}$. Since $Q_{\text {Heater }}=c \cdot m \cdot \Delta T \propto A$, the required amount of heat increases as the area of the heating zone becomes larger for the desired temperature gradient. $Q_{\text {Heater }}$ is the amount of heat, $c$ is the heat capacity, $m$ is the mass of the object, $A$ is the area of the heater and $\Delta T$ is the temperature gradient. In addition, the square of the input voltage is proportional to the amount of heat because $Q_{\text {Heater }} \propto V^{2} / R$, where $V$ is the input voltage, and $R$ is the resistance of the heater. Therefore, compared with the data in Figure $3 b$, this enlargement of the heater size required additional energy to raise its temperature, which required a higher input voltage to heat it to the target temperature. A cyclic heating test was conducted by repeatedly applying a DC bias to this large-area heater. This heater provided reliability, irrespective of the repetition of its on/off functions (Figure 4e). In the absence of the passivation layer to cover the surface of the AgNFs, 100 cycles of repetitive heating to $250{ }^{\circ} \mathrm{C}$ oxidized the AgNF surface in air (Supplementary Figures S5 and S6). A small peak at a binding energy of $529.4 \mathrm{eV}$ observed in the O 1s XPS spectrum after the repetitive heating test, as well as slightly broadened Ag peaks in the $\mathrm{Ag} 3 \mathrm{~d}$ spectrum, indicate the oxidation of $\mathrm{Ag}$. In addition, XRD also indicates the formation of $\mathrm{Ag}_{2} \mathrm{O}$ after this heating in air. As shown in the TEM image of Supplementary Figure S4, however, the thickness of this silver oxide layer was thinner than $\sim 1 \mathrm{~nm}$, and the heater could still operate without its failure. In addition, passivation layers can retard the oxidation of $\mathrm{Ag}$ in air (Figure 4e and Supplementary Figure S13). As a stretchable heater, random networks of AgNFs were formed on the PDMS sheet, and Figure $4 \mathrm{f}$ and Supplementary Movie S3 show that this sample can be stretched by up to $90 \%$ in tensile strain without significant degradation of the temperature. Supplementary Figure S16 presents the mechanical reliability of the AgNF heater against its bending or stretching. The temperature of this heater reduced slightly $\left(\Delta T / T_{0}: 27 \%\right)$ after 10000 cycles of repetitive stretching to $30 \%$ in strain. In addition, its temperature was negligibly downgraded against 3000 cycles of repetitive bending to $5 \mathrm{~mm}$ of the bending radius (bending-induced strain: $0.5 \%$ ).

\section{Demonstration of a wirelessly operated, stretchable, transparent heater}

Advances in wearable electronics and smart living are accelerating in a complementary manner, and the importance of wireless communications is increasing rapidly. For example, the extensive prevalence of smartphones enables the wireless operation of wearable, lightweight, portable electronic devices without any bulky components (for example, input-output units and controllers) that is integrated directly with these wearable devices. As an example, Figure 5 shows the wireless operating system of a transparent and flexible heater based on AgNF networks, with temperature control using a smartphone via Bluetooth. Figure 5a shows a schematic image of this wearable heater and its components for wireless operation. In this application, the flexible and stretchable heating film can be attached to human skin directly and connected to a wireless controller, which consists of a microcontroller unit (MCU), Bluetooth module, and lithium-polymer battery. In this case, the size of the wireless controller was $2.4 \times 3.2 \mathrm{~cm}^{2}$. Figure $5 \mathrm{~b}$ shows a circuit diagram, and a customdesigned circuit program was installed in the MCU to control the temperature (Supplementary Figure S17). In contrast with conventional heaters that require relatively large voltages and significant 
a Transparent, foldable and stretchable heater

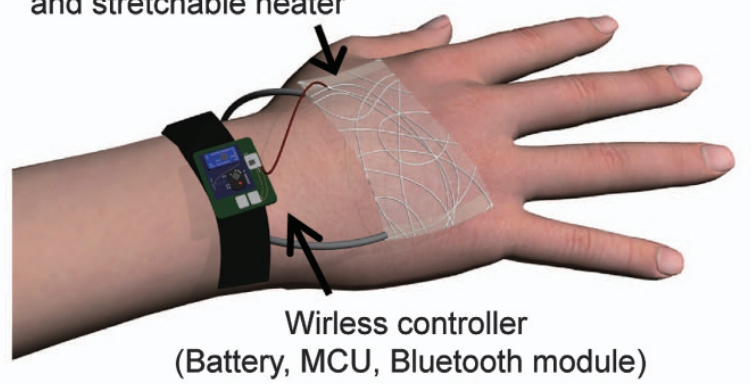

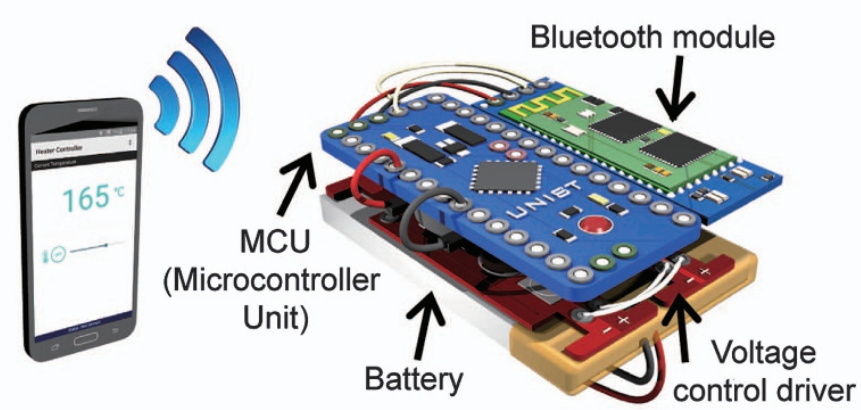

C
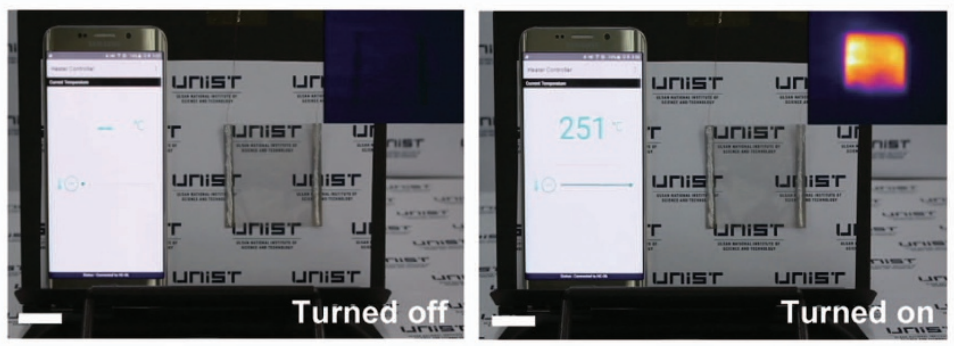

b

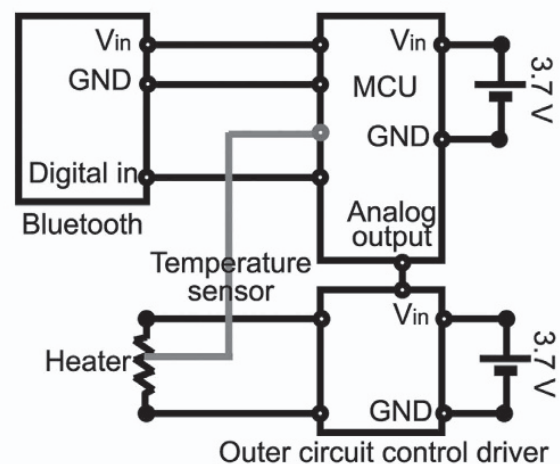

d

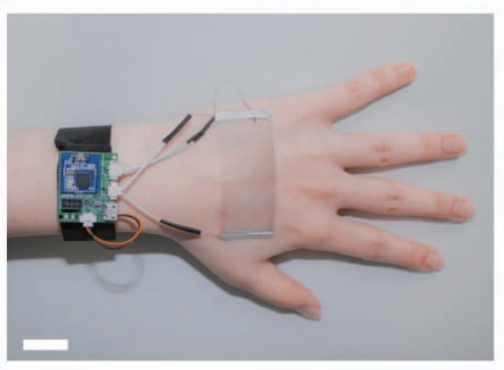

e

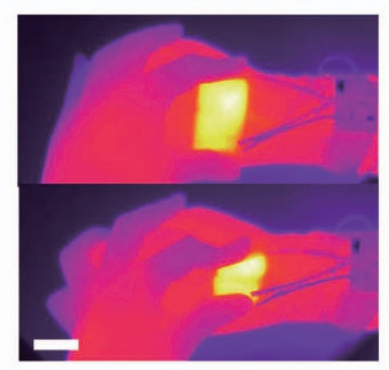

f

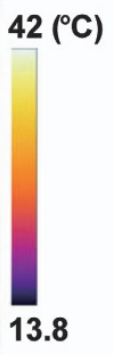

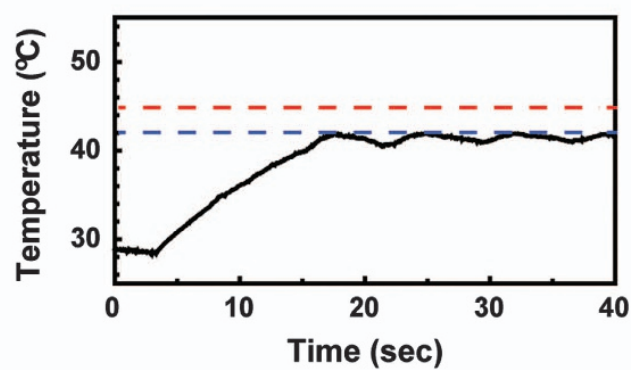

Figure 5 Applications for stretchable and transparent heaters that are based on AgNFs. (a) Schematic illustrations of wearable heaters with a controller unit (left) and wireless operation between a smartphone and the Bluetooth-integrated temperature controller circuits (right). (b) The corresponding circuit diagram of the temperature controller for the heater. (c) Screenshots from a movie in which the heater is being operated wirelessly a smartphone (left, turned off; right, turned on). The insets show the IR images of the heater. The scale bars represent $2 \mathrm{~cm}$. (d) A photograph of the stretchable, wearable heater with a wristband-type wireless operation device. The scale bars represent $2 \mathrm{~cm}$. (e) IR images of the stretchable wearable heater integrated with the wireless operating system. The scale bars represent $2 \mathrm{~cm}$. (f) The temperature as a function of time for the automatically programmed stretchable wearable heater. The blue dashed line indicates the target temperature, and the red dashed line indicates the limiting value of the low-temperature burn of human skin threshold.

power for their operations, the low $R_{\mathrm{s}}$ value and high power efficiency of the AgNF heater facilitate reductions in the sizes of the wireless controller and battery. A logical value of the input signal, which is associated with the target temperature, increases its corresponding DC voltage from 0 to $3.7 \mathrm{~V}$, which determines the amount of electric current flowing through the heater according to Joule's law. This logical value can be sent wirelessly to the heater via Bluetooth using a smartphone. Figure $5 c$ presents the wireless operation of this heater in real time (Supplementary Movie S4). The upper right insets show the corresponding IR images.

AgNF heaters can be used in various fields because of their low operating voltage, high heat output and ability to communicate wirelessly with smart devices. Figure $5 \mathrm{~d}$ shows photographs of a stretchable and wearable heater with a wristband-type wireless operating system using Bluetooth. The network of AgNFs was formed on the PDMS substrate for stretchability. The device is small enough $\left(2.4 \times 3.2 \mathrm{~cm}^{2}, 40 \mathrm{~g}\right)$ to mount on the human body, even on the skin, so that the user can wear it all the time. The AgNF heater showed stable operation in real time by wireless communication during mechanical deformation, such as compressive and tensile strain, indicating its great feasibility for use in healthcare applications (Figure 5e and Supplementary Movie S5). Furthermore, the device can be used for smart windows or warm clothes, because it can be fabricated regardless of the size. Figure $5 \mathrm{f}$ shows the temperature of the heater as a function of time. The blue, dashed line in the graph represents the target temperature $\left(42^{\circ} \mathrm{C}\right)$, and we confirmed that the temperature of the device remained constant. Furthermore, there is no concern about low-temperature burns because the temperature of the heater is programmed so that it cannot exceed the low-temperature 
burn limitation $\left(45^{\circ} \mathrm{C}\right)$, which is indicated by the red dashed line on the graph.

\section{CONCLUSION}

In conclusion, in this paper, we described the rapid production of transparent and stretchable electrodes using ultra-long AgNFs and proposed their use as large-area, wearable heaters that can be wirelessly operated. Continuous networks of long metal nanofibers can be produced directly on various flexible sheets as electrodes with large areas by using a roll-based electrospinning system for rapid production. This AgNF electrode exhibits remarkable optoelectronic properties and outstanding mechanical robustness against deformation, such as bending and stretching. Furthermore, this electrode can be used as a transparent and stretchable heater through the Joule heating of this film, and the temperature can exceed $250^{\circ} \mathrm{C}\left(600^{\circ} \mathrm{C}\right)$ on a polyimide sheet (a quartz substrate). Moreover, the temperature distribution of this heater during mechanical deformation is uniform due to its good flexibility and stretchability. Compared with conventional transparent heaters that are based on ITO or metal nanowires, the low $R_{\mathrm{s}}$ value of these AgNF networks enables relatively fast heating and cooling rates, as well as an enhanced power efficiency. Integration with a Bluetooth module and MCU demonstrates the potential for creating a portable, transparent heater with precise temperature control that is operated wirelessly via a smartphone. We believe that this approach presents a promising strategy for developing transparent and wearable electronic devices.

\section{CONFLICT OF INTEREST}

The authors declare no conflict of interest.

\section{ACKNOWLEDGEMENTS}

This work was supported by the Ministry of Science, ICT \& Future Planning and the Ministry of Trade, Industry and Energy (MOTIE) of Korea through the National Research Foundation (2016R1A2B3013592 and 2016R1A5A1009926), the Nano Material Technology Development Program (2015M3A7B4050308 and 2016M3A7B4910635), the Convergence Technology Development Program for Bionic Arm (NRF-2014M3C1B2048198), and the Pioneer Research Center Program (NRF-2014M3C1A3001208). Additionally, the authors gratefully received financial support through the Development Program of Manufacturing Technology for Flexible Electronics with High Performance (SC0970) funded by the Korea Institute of Machinery and Materials, and through the Development Program of Internet of Nature System (1.150090.01) funded by UNIST. JJ and BGH designed and performed the experiment, fabricated the devices and analyzed the data. SJ and EC contributed to the sample preparation and data analysis. BWA and WHC designed the wireless control circuit and conducted the wireless operation experiments. J-UP oversaw all research phases and revised the manuscript. All authors discussed and commented on the manuscript.

\section{PUBLISHER'S NOTE}

Springer Nature remains neutral with regard to jurisdictional claims in published maps and institutional affiliations.

1 Jang, S., Jang, H., Lee, Y., Suh, D., Baik, S., Hong, B. H. \& Ahn, J.-H. Flexible, transparent single-walled carbon nanotube transistors with graphene electrodes. Nanotechnology 21, 425201 (2010).

2 Choi, I., Jeong, H. Y., Jung, D. Y., Byun, M., Choi, C.-G., Hong, B. H., Choi, S.-Y. \& Lee, K. J. Laser-induced solid-phase doped graphene. ACS Nano 8, 7671-7677 (2014).

3 Kim, W.-K., Lee, S., Lee, D. H., Park, I. H., Bae, J. S., Lee, T. W., Kim, J.-Y., Park, J. H., Cho, Y. C., Cho, C. R. \& Jeong, S.-Y. Cu Mesh for flexible transparent conductive electrodes. Sci. Rep. 5, 10715 (2015).
4 Lee, H., Choi, T. K., Lee, Y. B., Cho, H. R., Ghaffari, R., Wang, L., Choi, H. J., Chung, T. D., Lu, N., Hyeon, T., Choi, S. H. \& Kim, D.-H. A graphene-based electrochemical device with thermoresponsive microneedles for diabetes monitoring and therapy. Nat. Nanotechnol. 11, 566-572 (2016).

5 Jung, M. W., Myung, S., Song, W., Kang, M.-A., Kim, S. H., Yang, C.-S., Lee, S. S., Lim, J., Park, C.-Y., Lee, J.-O. \& An, K.-S. Novel Fabrication of flexible graphene-based chemical sensors with heaters using soft lithographic patterning method. ACS Appl. Mater. Interfaces 6, 13319-13323 (2014).

6 An, B. W., Gwak, E.-J., Kim, K., Kim, Y.-C., Jang, J., Kim, J.-Y. \& Park, J.-U. Stretchable, transparent electrodes as wearable heaters using nanotrough networks of metallic glasses with superior mechanical properties and thermal stability. Nano Lett. 16, 471-478 (2016).

7 Choi, S., Park, J., Hyun, W., Kim, J. Kim, J., Lee, Y. B., Song, C., Hwang, H. J., Kim, J. H., Hyeon, T. \& Kim, D.-H. Stretchable heater using ligand-exchanged silver nanowire nanocomposite for wearable articular thermotherapy. ACS Nano $\mathbf{9}$, 6626-6633 (2015).

8 Kim, H., Gilmore, C. M., Piqué, A., Horwitz, J. S., Mattoussi, H., Murata, H., Kafafi, Z. H. \& Chrisey, D. B. Electrical, optical, and structural properties of indiumtin-oxide thin films for organic light-emitting devices. J. Appl. Phys. 86, 6451-6461 (1999).

9 Triambulo, R. E., Cheong, H.-G., Lee, G.-H., Yi, I.-S. \& Park, J.-W. A transparent conductive oxide electrode with highly enhanced flexibility achieved by controlled crystallinity by incorporating $\mathrm{Ag}$ nanoparticles on substrates. J. Alloys Compd. 620, 340-349 (2015).

10 Lee, C., Cuong, H. B., Jeong, S.-H. \& Lee, B.-T. Comparative study of group-II alloying effects on physical property of $\mathrm{ZnGaO}$ transparent conductive films prepared by RF magnetron sputtering. J. Alloys Compd. 645, 322-327 (2015).

11 Kang, J., Jang, Y., Kim, Y., Cho, S.-H., Suhr, J., Hong, B. H., Choi, J.-B. \& Byun, D. An Ag-grid/graphene hybrid structure for large-scale, transparent, flexible heaters. Nanoscale 7, 6567-6573 (2015).

12 Kang, J., Kim, H., Kim, K. S., Lee, S.-K., Bae, S., Ahn, J.-H., Kim, Y.-J., Choi, J.-B. \& Hong, B. H. High-performance graphene-based transparent flexible heaters. Nano Lett. 11, 5154-5158 (2011).

13 Li, Z., Xu, Z., Liu, Y., Wang, R. \& Gao, C. Multifunctional non-woven fabrics of interfused graphene fibres. Nat. Commun. 7, 13684 (2016).

14 Yoon, Y.-H., Song, J.-W., Kim, D., Kim, J., Park, J.-K., Oh, S.-K. \& Han, C.-S. Transparent film heater using single-walled carbon nanotubes. Adv. Mater. 19 4284-4287 (2007).

15 Janas, D. \& Koziol, K. K. Rapid electrothermal response of high-temperature carbon nanotube film heaters. Carbon 59, 457-463 (2013).

16 Hong, S., Lee, H., Lee, J., Kwon, J., Han, S., Suh, Y. D., Cho, H., Shin, J., Yeo, J. \& Ko, S. H. Highly stretchable and transparent metal nanowire heater for wearable electronics applications. Adv. Mater. 27, 4744-4751 (2015).

$17 \mathrm{Kim}$, T., Kim, Y. W., Lee, H. S., Kim, H., Yang, W. S. \& Suh, K. S. Uniformly interconnected silver-nanowire networks for transparent film heaters. Adv. Funct. Mater. 23, 1250-1255 (2013).

18 Jo, H. S., An, S., Lee, J.-G., Park, H. G., Al-Deyab, S. S., Yarin, A. L. \& Yoon, S. S. Highly flexible, stretchable, patternable, transparent copper fiber heater on a complex 3D surface. NPG Asia Mater. 9, e347 (2017)

19 Webb, R. C., Ma, Y., Krishnan, S., Li, Y., Yoon, S., Guo, X., Feng, X., Shi, Y., Seidel, M., Cho, N. H., Kurniawan, J., Ahad, J., Sheth, N., Kim, J., Vi, J. G. T., Darlington, T. Chang, K., Huang, W., Ayers, J., Gruebele, A., Pielak, R. M., Slepian, M. J., Huang, Y., Gorbach, A. M. \& Rogers, J. A. Epidermal devices for noninvasive, precise, and continuous mapping of macrovascular and microvascular blood flow. Sci. Adv. 1, e1500701 (2015).

20 Lee, H., Song, C., Hong, Y. S., Kim, M. S., Cho, H. R., Kang, T., Shin, K., Choi, S. H., Hyeon, T. \& Kim, D.-H. Wearable/disposable sweat-based glucose monitoring device with multistage transdermal drug delivery module. Sci. Adv. 3, e1601314 (2017).

21 Jang, H.-S., Jeon, S. K. \& Nahm, S. H. The manufacture of a transparent film heater by spinning multi-walled carbon nanotubes. Carbon 49, 111-116 (2011).

22 Webb, R. C., Bonifas, A. P., Behnaz, A., Zhang, Y., Yu, K. J., Cheng, H., Shi, M., Bian, Z., Liu, Z., Kim, Y.-S., Yeo, W.-H., Park, J. S., Song, J., Li, Y., Huang, Y., Gorbach, A. M. \& Rogers, J. A. Ultrathin conformal devices for precise and continuous thermal characterization of human skin. Nat. Mater. 12, 938-944 (2013).

23 Lee, J., Lee, P., Lee, H., Lee, D., Seob Lee, S. \& Hwan Ko, S. Very long Ag nanowire synthesis and its application in a highly transparent, conductive and flexible meta electrode touch panel. Nanoscale 4, 6408-6414 (2012).

24 Lee, M.-S., Lee, K., Kim, S.-Y., Lee, H., Park, J., Choi, K.-H., Kim, H.-K., Kim, D.-G., Lee, D.-Y., Nam, S. \& Park, J.-U. High-performance, transparent, and stretchable electrodes using graphene-metal nanowire hybrid structures. Nano Lett. 13, 2814-2821 (2013).

25 Nirmalraj, P. N., Bellew, A. T., Bell, A. P., Fairfield, J. A., McCarthy, E. K., O'Kelly, C., Pereira, L. F. C., Sorel, S., Morosan, D., Coleman, J. N., Ferreira, M. S. \& Boland, J. J. Manipulating connectivity and electrical conductivity in metallic nanowire networks. Nano Lett. 12, 5966-5971 (2012).

26 An, B. W., Hyun, B. G., Kim, S.-Y., Kim, M., Lee, M.-S., Lee, K., Koo, J. B., Chu, H. Y., Bae, B.-S. \& Park, J.-U. Stretchable and transparent electrodes using hybrid structures of graphene-metal nanotrough networks with high performances and ultimate uniformity. Nano Lett. 14, 6322-6328 (2014).

27 Wu, H., Kong, D., Ruan, Z., Hsu, P.-C., Wang, S., Yu, Z., Carney, T. J., Hu, L., Fan, S. \& Cui, Y. A transparent electrode based on a metal nanotrough network. Nat. Nanotechnol. 8, 421-425 (2013).

28 Wu, H., Hu, L., Rowell, M. W., Kong, D., Cha, J. J., McDonough, J. R., Zhu, J., Yang, Y., McGehee, M. D. \& Cui, Y. Electrospun metal nanofiber webs as high-performance transparent electrode. Nano Lett. 10, 4242-4248 (2010). 
29 Hsu, P.-C., Kong, D., Wang, S., Wang, H., Welch, A. J., Wu, H. \& Cui, Y. Electrolessly deposited electrospun metal nanowire transparent electrodes. J. Am. Chem. Soc. 136, 10593-10596 (2014).

30 Huang, Y., Bai, X., Zhou, M., Liao, S., Yu, Z., Wang, Y. \& Wu, H. Large-scale spinning of silver nanofibers as flexible and reliable conductors. Nano Lett. 16, 5846-5851 (2016).

31 Azuma, K., Sakajiri, K., Matsumoto, H., Kang, S., Watanabe, J. \& Tokita, M. Facile fabrication of transparent and conductive nanowire networks by wet chemical etching with an electrospun nanofiber mask template. Mater. Lett. 115, 187-189 (2014)

32 Bahiru Gebeyehu, M., Chang, Y.-H., Kelemework Abay, A., Chang, S.-Y., Lee, J.-Y., Wu, C.-M., Chiang, T.-C. \& Murakami, R.-I. Fabrication and characterization of continuous silver nanofiber/polyvinylpyrrolidone (AgNF/PVP) core-shell nanofibers using the coaxial electrospinning process. RSC Adv. 6, 54162-54168 (2016).

33 Jeong, J.-A., Kim, J. \& Kim, H.-K. Ag grid/ITO hybrid transparent electrodes prepared by inkjet printing. Sol. Energy Mater. Sol. Cells 95, 1974-1978 (2011).

34 Kang, M.-G., Kim, M.-S., Kim, J. \& Guo, L. J. Organic solar cells using nanoimprinted transparent metal electrodes. Adv. Mater. 20, 4408-4413 (2008).

35 Hong, S., Yeo, J., Kim, G., Kim, D., Lee, H., Kwon, J., Lee, H., Lee, P. \& Ko, S. H. Nonvacuum, maskless fabrication of a flexible metal grid transparent conductor by lowtemperature selective laser sintering of nanoparticle ink. ACS Nano 7, 5024-5031 (2013)

36 Cairns, D. R., Witte, R. P. II, Sparacin, D. K., Sachsman, S. M., Paine, D. C. \& Crawford, G. P. Strain-dependent electrical resistance of tin-doped indium oxide on polymer substrates. Appl. Phys. Lett. 76, 1425-1427 (2000).
37 Bae, S., Kim, H., Lee, Y., Xu, X., Park, J.-S., Zheng, Y., Balakrishnan, J., Lei, T., Ri Kim, H., Song, Y. I., Kim, Y.-J., Kim, K. S., Özyilmaz, B., Ahn, J.-H., Hong, B. H. \& lijima, S. Roll-to-roll production of 30-inch graphene films for transparent electrodes. Nat. Nanotechnol. 5, 574-578 (2010).

38 Soltanian, S., Rahmanian, R., Gholamkhass, B., Kiasari, N. M., Ko, F. \& Servati, P. Highly stretchable, sparse, metallized nanofiber webs as thin, transferrable transparent conductors. Adv. Energy Mater. 3, 1332-1337 (2013).

39 Li, P., Ma, J., Xu, H., Xue, X. \& Liu, Y. Highly stable copper wire/alumina/polyimide composite films for stretchable and transparent heaters. J. Mater. Chem. C 4, 3581-3591 (2016)

(c) (i) This work is licensed under a Creative Commons Attribution 4.0 International License. The images or other third party material in this article are included in the article's Creative Commons license, unless indicated otherwise in the credit line; if the material is not included under the Creative Commons license, users will need to obtain permission from the license holder to reproduce the material. To view a copy of this license, visit http:// creativecommons.org/licenses/by/4.0/

(C) The Author(s) 2017

Supplementary Information accompanies the paper on the NPG Asia Materials website (http://www.nature.com/am) 